Service social

\title{
Les conditions de vie des femmes séparées et les politiques sociales
}

\section{Madeleine Beaudry et Jean-Louis Gendron}

Volume 39, numéro 3, 1990

Familles recomposées après divorce

URI : https://id.erudit.org/iderudit/706502ar

DOI : https://doi.org/10.7202/706502ar

Aller au sommaire du numéro

\section{Éditeur(s)}

École de service social de l'Université Laval

ISSN

1708-1734 (numérique)

Découvrir la revue

Citer cet article

Beaudry, M. \& Gendron, J.-L. (1990). Les conditions de vie des femmes séparées et les politiques sociales. Service social, 39(3), 80-95.

https://doi.org/10.7202/706502ar
Résumé de l'article

L'objectif de cet article est de montrer dans quelle mesure les politiques sociales québécoises et canadiennes font une juste place aux femmes qui, dans la problématique des familles réorganisées, doivent assumer seules les responsabilités découlant de la monoparentalité. Dans une première partie, les auteurs présentent les conditions psychosociales des femmes séparées et soulignent toute l'importance du problème. Dans la deuxième partie de l'article, ils s'interrogent sur les mesures dont s'est dotée la société québécoise pour aider les femmes à assumer leurs responsabilités monoparentales. L'article conclut en reconnaissant une validité certaine aux efforts qui se font de plus en plus pour instaurer de telles mesures, mais il déplore l'absence dommageable d'une véritable politique familiale intégrée à la société québécoise. 
Madeleine Beaudry, professeure, École de service social, Université Laval.

Jean-Louis Gendron, professeur, École de service social, Université Laval.

\title{
Les conditions de vie des femmes séparées et les politiques sociales
}

\author{
Madeleine Beaudry \\ Jean-Louis Gendron
}

Au Québec, les familles réorganisées ${ }^{1}$ représentent $\pm 26 \%$ des familles (Statistique Canada, $n^{\circ}$ 94-109) et certaines études locales observent des taux plus élevés $( \pm 30 \%$ dans l'ensemble de la région urbaine de la ville de Québec avec des quartiers où la proportion de ces familles dépasse les 40 \% (Careau, Cloutier et Drolet, 1987). Bon nombre d'entre elles vivent en situation de monoparentalité où les mères assument souvent seules les responsabilités parentales. Dans une première partie de ce texte, nous tenterons de mettre en évidence l'importance des problèmes psychosociaux rencontrés. Au moment où souffle au Québec un vent de réforme dans les services sociaux, nous examinerons par la suite les faits saillants qui marquent l'engagement de l'État dans le soutien aux familles, en nous référant à la notion de politiques sociales et à la progression de ces politiques dans la société québécoise.

\section{Les conditions psychosociales des femmes séparées}

Le fait que les femmes s'occupent souvent seules de la garde des enfants à la suite d'une séparation n'est pas étranger aux problèmes qu'elles rencontrent. Selon le Conseil consultatif canadien sur la situation de la femme (1987: 29), le niveau de vie des femmes périclite à la suite d'un divorce, tandis que celui des hommes s'améliore. Ceux-ci ont presque le double de revenu net (comparativement à celui qu'ils avaient avant la séparation). Dans un cas sur deux, la 
monoparentalité pour les femmes signifie des revenus inférieurs au seuil officiel du faible revenu. Entre 1981 et 1985, leur situation s'est détériorée, leur taux de pauvreté passant de $53 \%$ à $60 \%$ (Lavigne, 1990 : 51). En 1988, on estime que $57 \%$ des familles monoparentales dirigées par des femmes avec des enfants dépendants sont pauvres (Ross, 1990 : 12). Cela est dû à différents facteurs, dont : la place des hommes évidemment plus avantageuse sur le marché du travail, le manque de barèmes objectifs pour fixer la pension alimentaire, l'octroi de pensions alimentaires souvent infimes et déductibles du revenu, rajustables selon l'augmentation des besoins de l'homme, le refus pour plusieurs hommes de payer ces pensions et, son corollaire, la faiblesse du système de perception des pensions, etc. Aux ÉtatsUnis, on observe le même appauvrissement des femmes séparées. D'après une recherche effectuée auprès de 228 personnes divorcées, Weitzman (1985) indique que le niveau de vie des femmes baisse de $73 \%$ après un divorce, tandis que celui des maris augmente de $42 \%$.

Au Québec, une enquête du ministère de la Justice effectuée en 1986 (Tremblay, $1987:$ 20-22) révèle que près de la moitié des mères qui ont la garde des enfants demeurent au foyer, sans aucun revenu d'emploi. Elles doivent donc vivre d'une maigre pension alimentaire, non indexée au coût de la vie et qui n'est octroyée par une ordonnance légale que pour $42 \%$ des couples avec enfants. Et encore faudrait-il que les femmes puissent recevoir cette pension. Les données de l'enquête révèlent que $63,5 \%$ des femmes touchent sans difficulté ce qui leur est dû, $13,8 \%$ ne le touchent qu'en partie et $22,7 \%$ ne le voient jamais ! Les hommes qui ne paient pas ne sont pas seulement les chômeurs et les assistés sociaux, car $83 \%$ des mauvais payeurs jouissent d'un revenu d'emploi.

Le Québec possède un service de perception alimentaire qui pourrait remédier à cette situation, mais seulement $20 \%$ des femmes admissibles y ont recours. Elles invoquent des raisons comme la durée et la complexité de la procédure, le manque présumé d'efficacité et la peur des représailles de leur ex-conjoint (Tremblay, 1987 : 20-22).

La pauvreté des femmes chefs de famille monoparentale est partagée par les enfants dont elles ont la garde. En 1988, au Canada, la probabilité d'être pauvre est six fois plus élevée pour un enfant qui grandit dans une famille monoparentale matricentrique que pour I'enfant qui grandit dans une famille avec ses deux parents; les taux de pauvreté s'élèvent respectivement à $64,6 \%$ et $10,2 \%$ (Freiler et Kitchen, 1990 : 47). Au Québec, 19,2 \% des enfants grandissent dans la pauvreté; $60 \%$ de ces enfants vivent dans des familles monoparentales (Bouchard, 1989 : 138). Aujourd'hui, on parle sans ambages de la 
"féminisation 》 de la pauvreté et de la "vulnérabilité » croissante même des personnes qui travaillent (Lesemann, 1989 : 114-119).

Les études sur les effets de la pauvreté indiquent assez clairement que la pauvreté accroît la probabilité que se manifestent des difficultés d'adaptation tant chez les enfants que chez les adultes (Bouchard, 1989; Bouchard et Perreault, 1983; Catalano et Dooley, 1983). Par ailleurs, la rupture conjugale constitue une source majeure de stress à laquelle sont souvent associés des problèmes psychologiques et émotionnels importants : sentiments d'échec, de honte, diminution de l'estime de soi (Bloom, 1978). Parmi les différentes situations de famille, les femmes divorcées, en particulier celles qui ont la garde de jeunes enfants, représentent le groupe qui a le plus haut taux d'anxiété et de dépression (Arendell, 1987).

Crago (1972), dans une recension de onze études menées durant une période de trente-cinq ans et portant sur l'incidence des maladies mentales et la situation matrimoniale, rapporte que les taux d'admission pour soins psychiatriques sont plus élevés chez les personnes séparées et divorcées que dans toute autre catégorie de situation matrimoniale. Dans une étude menée à l'hôpital Charles-LeMoyne de la Montérégie ${ }^{2}$ durant l'année administrative 1981-1982 (Julien, 1986), on observe que les personnes séparées et divorcées forment 17,0\% des utilisateurs de l'ensemble des services psychiatriques de l'hôpital et $19,0 \%$ des services à l'urgence, alors qu'elles ne représentent que $5 \%$ de la population. À ce chapitre, les femmes sont surreprésentées. Elles forment $59,8 \%$ des utilisateurs, alors que leur proportion est de $51,1 \%$ dans la population. Ces chiffres ne représentent pourtant qu'une petite partie des demandes de consultation. L'étude de Julien ne porte en effet que sur la clientèle ayant déjà utilisé des services spécialisés de psychiatrie. Elle ne comprend pas les personnes qui auraient consulté un médecin, un psychologue, un travailleur social ou tout autre professionnel de la santé.

En plus de faire face aux difficultés reliées à la pauvreté et à la séparation, les femmes "chefs de famille monoparentale " doivent composer avec la présence des enfants. Ces femmes doivent concilier leurs nouvelles responsabilités de parent unique et de pourvoyeur. Plusieurs ont un réseau social restreint et se sentent " enfermées dans un monde d'enfants " pour lesquels elles sont moins disponibles (Hetherington, 1976). Comparativement à ce qui est observé chez leur ex-conjoint, la probabilité de remariage des femmes est plus réduite. Aussi, plus elles auront de problèmes économiques, personnels et relationnels avec leur ex-conjoint (hostilité, manque d'appui, etc.), plus leurs enfants auront de la difficulté à s'adapter à la situation (Kalter et al., 1989). 
Les difficultés dans l'éducation des enfants associées aux problèmes économiques amènent souvent les femmes à consulter les services sociaux. Une étude visant à connaître la population du territoire de la Montérégie et les caractéristiques de la clientèle desservie par le Centre de services sociaux Richelieu couvrant ce territoire révèle des résultats pertinents (Oxman-Martinez, 1987).

En Montérégie, plus des trois quarts des parents seuls sont des femmes. Celles qui sont sur le marché du travail ne gagnent que $51 \%$ du salaire moyen des hommes. Une proportion de $36 \%$ des familles monoparentales soutenues par une femme ont un revenu inférieur au seuil de la pauvreté. Au Centre des services sociaux, la variable « monoparentalité " est associée de façon statistiquement significative aux signalements d'adolescents à la Direction de la protection de la jeunesse, aux demandes de services pour les adultes handicapés physiques et mentaux et pour les personnes âgées. Associée à la variable "propriété de moins de 20000 \$ ", la monoparentalité est aussi significativement associée au volume de signalements des enfants de 0 à 9 ans. Le fait d'être femme chef de famille monoparentale apparait donc comme un indicateur révélateur de besoins psychosociaux, particulièrement en ce qui concerne les problèmes familiaux et les difficultés économiques (Oxman-Martinez, $1987: 89$ ).

À tous ces problèmes, il ne faut pas oublier d'ajouter l'importance de la violence conjugale. Au Canada, l'estimation la plus souvent retenue est celle de McLeod (1980) selon laquelle une femme sur dix serait violentée. On peut supposer que le même rapport existe pour le Québec (Paquet-Deehy et al., 1985). Selon un document du ministère de la Santé et des Services sociaux (MSSS, 1985 : 10) portant sur la politique d'aide aux femmes battues, on estime à plus de 300000 le nombre de femmes victimes de violence. Au moment du divorce, ces femmes (et leurs enfants) ont besoin de protection. Pour certaines, il est essentiel d'assurer leur sécurité en coupant tout contact avec leur ex-conjoint abusif; dans ces cas, la garde exclusive $s^{\prime}$ impose. Mais du même coup, ces femmes se retrouvent à assumer seules toutes les responsabilités parentales.

II ne fait plus de doute que la pauvreté et l'ensemble des problèmes psychosociaux qui lui sont associés pèsent lourd sur les épaules des femmes. Depuis le début de la colonie où la garde et l'entretien des enfants étaient perçus comme une responsabilité privée, exclusivement réservée aux femmes et où l'État prétendait n'avoir aucun rôle à jouer, des changements se sont graduellement opérés, mettant en évidence l'aspect sociopolitique du problème. La deuxième partie de ce texte tentera de dégager ces principaux changements. 


\section{Les politiques sociales}

C'est en passant par l'histoire des politiques sociales que notre article tentera de déceler ces changements et d'en mesurer l'importance relative. Les politiques sociales sont le point de rencontre entre les personnes et les actions collectives entreprises pour promouvoir le bien-être de l'ensemble d'une population donnée (Beaudoin, 1987).

En effet, même si on les oppose parfois aux politiques économiques, culturelles, énergétiques ou autres, les politiques sociales n'en sont pas moins une catégorie, tout au plus un peu spéciale, de ce que l'on appelle habituellement les politiques publiques, c'est-à-dire " ces processus de régulation par lesquels l'État régit l'allocation des ressources au sein d'une collectivité » (Landry et Duchesneau, 1989).

Ainsi comprises, les politiques sociales sont perçues comme des moyens d'action, parmi d'autres, dont une société dispose pour atteindre ses objectifs. Or, ce qui caractérise les politiques sociales parmi l'ensemble des politiques publiques en général, c'est qu'elles sont centrées sur les personnes. Par le truchement des politiques sociales, ce sont les personnes, en effet, qui deviennent objet de régulation. Comme l'a écrit Vincent Lemieux, "les personnes sont les centres déterminants ou déterminés des ensembles de moyens d'action que visent les politiques sociales. On peut interpréter ainsi les politiques dans le secteur de la santé, des services sociaux, de la maind'œuvre, de la famille, de la vieillesse, etc. » (Lemieux, 1989).

II s'agit donc bien, lorsqu'on parle de politiques sociales, de processus de régulation visant à réduire l'écart entre des situations observées et des idéaux sociaux que certains acteurs veulent actualiser au sein de la société. Dans cet article, il sera question des politiques sociales ayant trait à la régulation du rôle social de la famille. Plus précisément, nous posons le problème des politiques sociales visant les familles réorganisées, telles que définies en introduction, et tout particulièrement ces familles monoparentales où une femme doit jouer seule la plupart des rôles parentaux.

Quelles sont donc les mesures dont s'est dotée notre société pour aider les femmes à assumer leurs responsabilités monoparentales ? Cette question est complexe puisque, d'une part, ces mesures sont englobées dans des lois plus générales couvrant l'ensemble des familles. D'autre part, ces mesures touchent plusieurs secteurs de l'organisation sociale : les services de garde, le soutien économique aux familles, le monde du travail et le droit familial'. Nous fournissons en annexe une liste exhaustive des principales étapes et lois qui marquent l'évolution historique de la situation pour chacun de ces secteurs. 


\section{Les services de garde}

La participation des gouvernements dans les services de garde pour les enfants (garderie) rappelle l'hésitation de l'État à se reconnaître des responsabilités dans un domaine jusque-là considéré comme privé et exclusivement féminin.

C'est en 1942, en effet, et avec une participation mitigée de la part du Québec, que le gouvernement fédéral et quelques provinces mettent sur pied des garderies pour les enfants dont la mère occupe un emploi jugé essentiel à l'effort de guerre. Puis, la guerre étant terminée et le Québec se montrant pendant longtemps réticent, il faudra attendre trente ans avant que la société québécoise ne s'engage, en 1974 avec le plan Bacon, dans le véritable démarrage des garderies à but non lucratif.

Par la suite, cependant, les choses s'accélèrent rapidement avec la Loi sur les services de garde à l'enfance (1979), la création de l'Office des services de garde à l'enfance (1980), l'adoption d'une politique concernant la garde en milieu familial (1984) et la modification de la loi québécoise sur les services de garde à l'enfance (1989).

En 1990, revers de I'histoire, c'est le gouvernement fédéral qui tarde à investir dans les soins de garde les cinq milliards de dollars promis en 1987 !

\section{Le soutien économique aux familles}

C'est dans l'esprit d'une lutte à la pauvreté que les premières mesures sociales seront mises de l'avant par les gouvernements du début du siècle et plus particulièrement par le gouvernement fédéral qui, en 1917, adopte un programme permettant des crédits d'impôt pour personnes à charge. II faudra cependant attendre jusqu'en 1945 le programme fédéral des allocations familiales et jusqu'en 1967 le programme québécois complémentaire aux allocations familiales. Ce dossier comprendra d'ailleurs l'abolition des exemptions d'impôt accordées jusqu'alors aux parents d'enfants de moins de seize ans. Ces dernières seront rétablies en 1985.

Suivront, dans les années 70 , le programme québécois de l'aide sociale, le programme fédéral des crédits d'impôt pour enfants de moins de 18 ans et les programmes de supplément du revenu et d'allocation de disponibilité. Dans les années 80 , la majoration des allocations de naissance constitue une mesure importante, pendant que les nouvelles taxes (TPS et TVQ) touchant plusieurs produits et services de base font planer sur le soutien économique aux familles une donnée nouvelle, importante et largement imprévisible dans ses conséquences. 


\section{Le monde du travail}

Depuis le début des années 70, l'aide à la parentalité, sous des dehors humanitaires, paraît bien répondre aussi à des nécessités économiques liées aux besoins de consommation et aux pressions politiques qui s'ensuivent. La décision des couples de faire carrière, tant pour favoriser l'égalité des sexes que pour subvenir à des besoins familiaux nouveaux, semble ici forcer l'État à adopter des politiques sociales de ce type.

Le gouvernement fédéral, en 1971, décide d'accorder des prestations de maternité dans le cadre du programme d'assurancechômage. II reconnaîtra aussi à la mère le droit de reprendre le travail après son congé de maternité. Quelques années plus tard, en 1979, le gouvernement québécois adoptera le programme fédéral de 1971 et créera même un programme d'allocation de maternité complémentaire.

Puis, au cours de la dernière décennie s'ajoutent : la reconnaissance du congé d'adoption, divers programmes visant à répondre aux besoins particuliers des femmes qui retournent sur le marché du travail après plusieurs années d'absence, l'introduction du congé parental pouvant être partagé entre les deux parents et différentes mesures dont l'importance reste à mesurer, telles ces " tables régionales d'accueil et de référence » que gère Travail-Québec.

\section{Le droit familial}

L'ancien Code civil reflétait le modèle de la famille traditionnelle où la femme était d'abord une mère et une épouse qui devait assurer les services de la vie quotidienne, pendant que le père pourvoyeur y détenait l'autorité. Ce code ne reconnaissait à la femme mariée aucune identité propre, ni juridique, ni sociale, ni économique. Les changements survenus à partir des années 60 dans le droit familial ont donné forme à un nouveau modèle, plus égalitaire.

En 1964, en effet, le Code civil est modifié et on y reconnaît la capacité juridique des femmes mariées. En 1970, l'adoption du régime légal de la société d'acquêts consacre le principe du partage des biens acquis pendant le mariage, alors qu'en 1979 est rendue officielle la Loi sur la protection de la jeunesse.

La dernière décennie, quant à elle, est fortement marquée par plusieurs changements importants du droit familial : réformes $\mathrm{du}$ Code civil et reconnaissance de l'égalité des époux quant au droit de garde, d'éducation et de surveillance des enfants; création d'un service de perception des pensions alimentaires; nouvelle loi fédérale où la garde partagée est reconnue pour la première fois; partage du 
patrimoine au moment de la séparation; amendements à la Loi sur la protection de la jeunesse.

\section{Conclusion}

Services de garde, soutien économique aux familles, monde du travail et droit familial, ces quatre catégories permettent de résumer l'ensemble des mesures dont s'est dotée la société québécoise pour aider les femmes à assumer leurs responsabilités parentales. Elles passent toutefois sous silence une autre loi fondamentalement importante : la Charte des droits et libertés. En établissant, en effet, l'égalité juridique des personnes, la Charte des droits et libertés, adoptée en 1983, se pose comme ultime justification de l'égalité père-mère dans la responsabilité parentale. II faut donc prévoir que cette charte sera, dans les années à venir, la pièce fondamentale sur laquelle se poseront les politiques sociales s'adressant spécifiquement aux femmes séparées, et surtout la coordination des différentes mesures qui en découleront.

L'analyse qui précède permet en effet de constater que cet ensemble de lois et de mesures très hétéroclites et encore mal intégrées les unes aux autres semblent cependant se diriger lentement mais sûrement vers une politique sociale adaptée aux besoins des femmes séparées élevant seules leurs enfants.

Ce qui manque le plus, $c^{\prime}$ est une véritable coordination entre ces différents éléments disparates, de façon à favoriser les personnes qui assument ces responsabilités parentales.

$C^{\prime}$ est d'ailleurs ce qui ressort d'un grand nombre de rapports rédigés dans les années 80 et dont le résumé permettra de conclure cet article.

Le Document de consultation sur la politique familiale est le premier texte produit par un gouvernement en vue de l'adoption d'une politique familiale; le rapport du Conseil du statut de la femme, Pour les québécoises : égalité et indépendance... 5 ans après, évalue les suites données par le gouvernement aux revendications féminines; Participation et intégration. Les femmes, le travail et l'argent, du Conseil consultatif canadien sur la situation de la femme, formule lui aussi ses recommandations; les deux volumes du Comité de la consultation sur la politique familiale du Québec (Le soutien collectif recommandé pour les parents et Le soutien collectif réclamé pour les familles québécoises) proposent les grandes orientations d'une politique familiale; le Mémoire présenté par le Conseil du statut de la femme à la Commission sur l'avenir politique et constitutionnel du Québec y souligne combien le partage des compétences entre les deux paliers 
de gouvernement affecte la situation des femmes; et, finalement, cette pièce maîtresse des changements sociaux en cours, Une réforme axée sur le citoyen, fait appel aux transformations radicales de la famille pour justifier les mesures qu'elle propose. Toutes ces études, tous ces rapports critiquent la politique gouvernementale actuelle et formulent leurs recommandations.

Ces recommandations prennent plusieurs teintes: revoir les pensions alimentaires, élaborer une politique plus cohérente en ce qui concerne la violence familiale, recourir aux services de médiation, faire participer davantage les hommes dans les soins et l'éducation des enfants, faciliter l'accessibilité aux services de garde, etc.

Au cœur de ces multiples recommandations, omniprésente et réclamée par tous, c'est une politique familiale, intégrée au cadre de la société québécoise et gérée par le gouvernement du Québec, qui semble manquer le plus. De cette politique devrait découler une reconnaissance que la garde des enfants ne relève plus de la seule responsabilité des femmes, que les politiques sociales dans leur fonction régulatrice pourraient davantage en tenir compte et que les conditions de vie des femmes séparées y gagneraient largement.

\section{Annexe}

Liste des principaux éléments historiques, législatifs et politiques ayant trait aux politiques sociales concernant la situation des femmes séparées.

\section{A) Les services de garde ${ }^{3}$}

1942 : Le gouvernement fédéral et quelques provinces mettent sur pied des garderies pour les enfants dont la mère occupe un emploi jugé essentiel à l'effort de guerre. Cependant, le Québec participe peu.

1972 : Le Québec n'a pas encore de normes pour assurer la garde des enfants. Il accepte cependant les frais de garde comme déductions fiscales.

1974 : Plan Bacon : c'est le démarrage des garderies à but non lucratif. Ce rapport prépare la Loi sur les services de garde. II prévoit le remboursement d'une partie des frais de garde engagés par les parents à bas revenus.

1979 : Loi sur les services de garde à l'enfance.

1980 : Création de l'Office des services de garde à l'enfance (Québec). Les services deviennent accessibles aux familles qui en ont le plus besoin et une aide financière est accordée aux parents à faibles revenus. 
1983 : L'Office des services de garde soumet un document intitulé Situation actuelle et perspectives de développement des services de garde à l'enfance au Québec (1983-1988). Ce plan est considéré comme un engagement ferme à trouver des ressources financières et humaines nécessaires au développement des garderies.

1984 : Adoption d'une politique concernant la garde en milieu familial.

1985 : Pour l'ensemble du Québec, le nombre de places disponibles en garderie ne répond encore qu'à $15 \%$ des besoins.

1987 : Même s'il y a eu d'énormes progrès, le plan de l'Office de garde n'a jamais été adopté et les places manquent toujours en garderie. Le projet de loi fédéral sur les garderies est remis « à plus tard »... L'accord constitutionnel du lac Meech et la question du libre-échange occupent toute la place au cabinet des ministres (Presse canadienne, 1987). En décembre, le gouvernement fédéral annonce un investissement de cinq milliards de dollars pour les prochaines années.

1989 : La Loi sur les services de garde à l'enfance (L.R.Q., c. S-4.1) est modifiée de façon à structurer l'organisation des services de garde en milieu familial.

1990 : Les investissements du gouvernement fédéral tardent à venir sous prétexte qu'ils seront répartis sur un plus grand nombre d'années.

\section{B) Le soutien économique aux familles}

1917 : Programme canadien permettant des exemptions d'impôt pour personnes à charge.

1945 : Programme fédéral des allocations familiales.

1967 : Programme québécois complémentaire aux allocations familiales (abolition des exemptions accordées jusqu'alors aux parents d'enfants de moins de 16 ans).

1970 : Entrée en vigueur du programme québécois de l'aide sociale sous la forme qu'on lui connaît aujourd'hui, sauf quelques modifications dont l'indexation (1983).

1974 : Programme québécois de développement des garderies (voir service de garde plus haut).

1978 : Programme fédéral, accepté par le Québec. On accorde un crédit d'impôts pour enfants de moins de 18 ans. C'est un programme sélectif qui permet aux familles les moins fortunées d'obtenir une compensation plus importante.

1979 : Programme québécois sur le supplément du revenu de travail. Ce programme complète le programme de l'aide sociale et 
incite les personnes les moins biens rémunérées à conserver leur emploi.

1981 : Programme québécois accordant des allocations de disponibilité. On reconnaît les tâches parentales exercées auprès des jeunes enfants comme service rendu à la collectivité. Les primes sont versées à la personne qui reçoit les allocations familiales (ordinairement la mère), à condition que l'un des parents ne déduise pas les frais de garde sur sa déclaration des revenus.

1985 : Rétablissement du programme provincial des exemptions pour personnes à charge.

1988 : Abolition des allocations familiales de base (Québec). Majoration des allocations de naissance jusqu'à 3000 \$ pour le troisième enfant et les suivants. Ajout d'allocations pour jeunes de moins de 6 ans. Majoration des déductions pour frais de garde. Réduction d'impôt pour les familles à faible et moyen revenu.

1990 : Selon les statistiques fiscales de 1988, la première phase de la réforme fiscale instaurée par le ministre des finances, $M$. Wilson, démontre que les contribuables dont le revenu est inférieur à $15000 \$$ ont versé $59 \%$ moins de taxes dans l'année; ceux qui bénéficient d'un revenu de $50000 \$$ et plus ont pour leur part versé $25 \%$ plus de taxes. La note des contribuables à revenu moyen (30 $000 \$$ à $50000 \$$ ) a grimpé quant à elle de $3 \%$. Par ailleurs, les crédits consacrés à de nouveaux engagements pour les logements sociaux sont réduits de $15 \%$.

1991 : Nouvelle taxe : la TPS. Des produits de base (comme la nourriture en petites portions et les vêtements) sont taxés. De plus, la coupure de $15 \%$ sur les logements sociaux se poursuivra jusqu'en 1995-1996.

\section{C) Le monde du travail}

1971 : Le gouvernement fédéral accorde des prestations de maternité dans le cadre du programme d'assurance-chômage. Il accorde à la mère le droit de reprendre le travail après son congé.

1979 : Le gouvernement québécois adopte le programme fédéral de 1971 et crée un programme d'allocation de maternité complémentaire.

1981 : Loi sur les normes du travail. On accorde des congés de maternité et la possibilité de retrait préventif pour les employées de l'État.

1983 : Le Programme québécois accorde à la travailleuse qui adopte un enfant les prestations accordées à la mère en vertu du programme d'assurance-chômage. La Loi sur les normes du travail ne reconnaît toutefois pas le congé de paternité. Elle 
accorde au père deux jours d'absence non rémunérés pour la naissance d'un enfant. De plus, elle ne protège pas bien les travailleuses à temps partiel.

1985-: Divers programmes sont mis sur pied pour répondre aux be-

1987 soins particuliers des femmes qui retournent sur le marché du travail après plusieurs années d'absence. Tables régionales d'accueil et de référence des bureaux de Travail-Québec, Transition-Travail, SEMO, etc. (Toutefois, ces programmes n'atteignent pas suffisamment de femmes.)

1990 : Réforme fédérale de l'assurance-chômage. Ottawa ajoute dix semaines de prestations parentales aux dix-sept semaines de prestations de maternité.

1990 : Loi provinciale modifiant la Loi sur les normes du travail et d'autres dispositions législatives (projet de loi 97). Québec introduit un congé parental de 34 semaines qui peut être partagé entre les deux parents. Toutefois, les prestations parentales seront gérées par le programme fédéral de l'assurancechômage, ce qui limite grandement la portée de cette mesure.

\section{D) Le droit familial}

1964 : Modification au Code civil. Reconnaissance de la capacité juridique des femmes mariées.

1970 : Adoption du régime légal de la société d'acquêts qui consacre le principe du partage des biens acquis pendant le mariage.

1979 : Loi sur la protection de la jeunesse.

1980 : Loi instituant un nouveau Code civil et, partant, réforme du droit de la famille (loi 89). On reconnaît l'égalité des époux dans le mariage, leur droit de garde, d'éducation et de surveillance.

1981 : Le Québec instaure un service de perception des pensions alimentaires, mais les femmes doivent souvent entreprendre des poursuites judiciaires pour se faire payer.

1984 : Loi 60 (modifications apportées à la Loi sur la protection de la jeunesse).

1985 : Nouvelle loi fédérale sur le divorce. La garde partagée (ou conjointe) y apparaît pour la première fois. Malgré la volonté explicite des tribunaux de contribuer à maintenir pour l'enfant la relation avec ses deux parents, la législation canadienne $\mathrm{n}^{\prime}$ accorde pas une priorité favorable à la garde conjointe. De plus, les juges ne peuvent obliger certains couples à se soumettre à la médiation avant que le tribunal ne règle le litige.

1989 : Loi modifiant le Code civil du Québec et d'autres dispositions législatives afin de favoriser l'égalité économique des époux. Cette loi touche particulièrement le partage du patrimoine fa- 
milial au moment de la séparation des époux. Ce patrimoine devra désormais être divisé à parts égales, entre les époux ou entre l'époux survivant et les héritiers, selon le cas.

Comme nous pouvons le constater, cet ensemble de lois ou de mesures touchent de manière plus ou moins directe l'organisation de la garde des enfants. II n'est pas évident toutefois que ces lois et mesures fassent l'objet d'une véritable coordination. C'est ce qui ressort d'un grand nombre de rapports rédigés dans les années $80 \mathrm{dont}$ voici un bref résumé.

1984 : Document de consultation sur la politique familiale, Québec. Premier document produit par un gouvernement en vue de I'adoption d'une politique familiale.

1984 : Pour les Québécoises : égalité et indépendance... 5 ans après, Conseil du statut de la femme. Ce rapport évalue les suites données par le gouvernement aux revendications des femmes.

1985 : Oui, mais... présente de manière plus succincte et sous forme de tableaux les progrès réalisés et les principales recommandations.

1987 : Participation et intégration. Les femmes, le travail et l'argent, Conseil consultatif canadien sur la situation de la femme. Dans le chapitre sur le mariage et l'argent, on fait état des rapports financiers entre les époux et ex-époux et on formule des recommandations.

1987 : Le soutien collectif réclamé pour les familles québécoises, Comité de la consultation sur la politique familiale, Québec. Le rapport contient deux volumes : "Le soutien collectif réclamé pour les familles québécoises" et "Le soutien collectif recommandé pour les parents ». Ce dernier volume définit les grandes orientations pour une politique familiale, fait état de l'ensemble des mesures adoptées par les divers paliers de gouvernement et formule un ensemble de recommandations.

1990 : Mémoire présenté par le Conseil du statut de la femme à la Commission sur l'avenir politique et constitutionnel du Québec. Le Conseil souligne que le partage des compétences entre les deux paliers de gouvernement affecte les femmes, en matière de conditions féminines, dans les secteurs suivants : le travail, le droit de la famille, la sécurité du revenu, les dépenses fédérales reliées aux services de garde, l'avortement et l'immigration. La nécessité d'intégration des différents aspects $d^{\prime}$ une politique de la condition féminine l'incite donc à favoriser une présence exclusive du Québec dans ces secteurs.

1990 : Projet de loi 120. Le ministre Côté propose une nouvelle réforme des services de santé et des services sociaux. II fait appel 
à divers motifs pour appuyer la réforme, en particulier aux problèmes de santé et de bien-être qu'entraîne la transformation radicale de la famille (Une réforme axée sur le citoyen, gouvernement du Québec, p. 8). Pourtant, les services à la famille ne font pas partie des grandes priorités retenues, sinon par le moyen de l'intervention auprès des jeunes.

\section{Notes}

${ }^{1}$ La notion de réorganisation familiale renvoie aux changements qui surviennent dans l'organisation de la famille après la séparation des parents.

${ }^{2}$ Sur la rive sud de Montréal.

${ }^{3}$ Le lecteur particulièrement intéressé par ce sujet pourra consulter le petit livre passionnant de Ghislaine Desjardins (1991), Faire garder ses enfants au Québec... Une histoire toujours en marche, Québec : Les Publications du Québec.

\section{Références bibliographiques}

ARENDELL, T.J. (1987). "Women and the economics of divorce in the contemporary United States ", Journal of Women in Culture and Society, vol. 13, $n^{\circ} 1$.

Association des centres de services sociaux du Québec (1984). Vers un bilan. Montréal.

Beaudoin, A. (1988). Services sociaux à l'enfance, à la famille, aux personnes âgées et dans le domaine de la santé. L'état de la situation. Laboratoire de recherche, École de service social, Université Laval.

BeAudoin, A. (1987a). Le social et la politique sociale : domaines et orientation d'analyse. Texte inédit.

BEAUDOIN, A. (1987b). Le champ des services sociaux dans la politique sociale au Québec. Commission d'enquête sur les services de santé et les services sociaux. Québec : Les Publications du Québec.

BLOOM, B.L. (1978). "Marital disruption as stressor", dans D. Forgays (éd.). Environmental Influences and Strategies in Primary Prevention. Hanover \& London : University Press of New England.

BOUCHARD, C. (1989). "Lutter contre la pauvreté ou ses effets ? Les programmes d'intervention précoce ", Santé mentale au Québec, vol. XIV, n 2 : 138-149.

Bouchard, C. et R. Perreault (1983). "Fermeture d'usine et santé : Analyses de séries chronologiques chez les femmes de 30 à 39 ans de la région de Septîles-Port-Cartier ", Revue canadienne de santé mentale communautaire, vol. 1 : 101-107.

Careau, R., R. Cloutier et J. Drolet (1987). La garde de l'enfant après la séparation des parents. Communication présentée au Congrès annuel de la Corporation professionnelle des psychologues du Québec. Québec, mai.

Caron-Gaulin, H. (1985). "Une pratique en mutation ", Intervention, no 72 : 23-34. 
CATAlANO, R. et D. Dooley (1983). "The health effects of economic instability : A test of the economic stress hypothesis. Journal of Health and Social Behavior, $\mathrm{n}^{\circ} 24: 46-60$.

Comité de la consultation sur la politique familiale (1987). Le soutien collectif réclamé pour les familles québécoises et Le soutien collectif recommandé pour les parents québécois. Québec : Gouvernement du Québec.

Conseil consultatif canadien sur la situation de la femme (1987). Participation et intégration. Les femmes, le travail et l'argent. Ottawa.

Conseil du statut de la femme (1985). Oui, mais. . Le bilan des activités gouvernementales en matière de condition féminine est-il positif ? Bibliothèque nationale du Québec.

Conseil du statut de la femme (1991). Mémoire présenté à la Commission sur I'avenir politique et constitutionnel du Québec. Québec.

Conseil du statut de la femme (1984). Pour les Québécoises : égalité et indépendance... 5 ans après. Québec.

Crago, M.A. (1972). "Psychopathology in married couples", Psychological Bulletin, $\mathrm{n}^{\circ} 77: 11-128$.

Desjardins, G. (1991). Faire garder ses enfants au Québec... Une histoire toujours en marche. Québec : Les Publications du Québec.

DorÉ, G. et L. Gaudreau (1989). " Politique sociale, politique partisane et profession ", Service social, vol. 38, nos 2-3 : 246-262.

DUCHESNeAu, P. et R. LANDRY (1989). "À qui profitent les lois de la mission sociale?", Service social, vol. 38, nos 2-3 : 196-219.

DUPLANTIE, J.-P. (1984). "Pour un véritable bilan de la pratique sociale dans les CSS ", Intervention, $\mathrm{n}^{\circ} 68: 2-12$.

FORTIN, A. (1987). Histoire de familles et de réseaux : La sociabilité au Québec d'hier à demain. Montréal : Les Éditions Saint-Martin.

Freiler, C. et B. KitCHen (1990). "Family portrait », Perception, vol. 14, n 2 : 46-52.

Gouvernement du Québec (1984). Document de consultation sur la politique familiale. Québec.

Gouvernement du Québec, ministère de la Santé et des Services sociaux (1990). Une réforme axée sur le citoyen.

Gouvernement du Québec (1988). Rapport de la commission d'enquête sur les services de santé et les services sociaux. Québec : Les Publications du Québec.

Hetherington, E.M., M. Cox, et R. Cox (1976). "Divorced Fathers », The Family Coordinator, $\mathrm{n}^{\circ} 25: 417-428$.

JULIEN, M. (1986). La clientèle des services psychiatriques pour adultes de l'hôpital Charles-LeMoyne. Saint-Lambert : DSC Charles-LeMoyne.

Kalter, N., A. Kloner, S. SChreier et K. Okla (1989). "Predictors of children's postdivorce adjustment ", American Journal of Orthopsychiatry, vol. 59, $\mathrm{n}^{\circ} 4: 605-618$.

LAFOREST, J. (1984). "La nature du service social », Intervention, $\mathrm{n}^{\circ} 68: 22-32$.

LAPOINTE, M. (1985). "Les enjeux professionnels du transfert CSS/CLSC pour les travailleurs sociaux ", Intervention, $\mathrm{n}^{\circ} 71$.

Lavigne, M. (1990). " D'une décennie des femmes à l'autre ", Perception, vol. 14, $\mathrm{n}^{\circ} 2$ : 50-52. 
LemieuX, V. (1989). "Le pouvoir dans la réalisation des politiques sociales", Service social, vol. $38, \mathrm{n}^{\text {os }} 2-3: 179-195$.

LESEMANN, F. (1989). "Les nouveaux visages de la pauvreté au Québec ", Santé mentale au Québec, vol. XIV, nº 2 : 114-119.

LINDSAY, J. et G. MARTIN (1984). "Le partage des responsabilités entre les Centres de services sociaux et les Centres locaux de services communautaires - impact pour la pratique et pour la formation ", Revue canadienne de service social, Ottawa.

MAYER, R. et L. Groulx (1987). Synthèse critique de la littérature sur l'évolution des services sociaux au Québec depuis 1960. Commission d'enquête sur les services de santé et les services sociaux. Québec : Les Publications du Québec.

MCLOAD, L. et A. CADIEUX (1980). La femme battue au Canada : un cercle vicieux. Conseil consultatif canadien sur la situation de la femme. Ottawa.

MSSS (1985). Une politique d'aide aux femmes violentées. Québec : Gouvernement du Québec.

OXMAN-MARTINeZ, J. (1987). Indicateurs psychosociaux de vulnérabilité : la " monoparentalité ", une variable clé. Étude sur la distribution territoriale de la nature et du volume de la demande de services au CSSR (région de la Montérégie). Direction des services professionnels. Centre des services sociaux Richelieu.

Paquet-Deehy, A., M. Rinfret-Raynor et G. Larouche (1985). "Violence familiale : un colloque. Évolution actuelle au plan de la recherche, de l'intervention et de la formation ", Revue canadienne de service social : 315-330.

Poulin, M. (1982). "Prospective pour la gestion des services sociaux des années quatre-vingt au Québec ", Service social, vol. 31, n 1.

REDJEB, B. (1984). "Le partage des responsabilités CSS/CLSC : de l'évidence à l'équivoque ", Revue canadienne de service social, Ottawa.

RobichaUd, J.B. (1979). "Dilemmes actuels de la profession ", Intervention, $n^{\circ} 56: 26-47$.

Ross, D.P. (1990). " ... And the poor got poorer ", Perception, vol. 14, $\mathrm{n}^{\circ} 2$ : 11-15.

SIMARD, M. et J. VACHON (1987). La politique de placement d'enfants : étude d'implantation dans deux régions du Québec. Commission d'enquête sur les services de santé et les services sociaux. Québec : Les Publications du Québec.

Statistique Canada (1986). Statistiques sur la population. Publication n ${ }^{\circ}$ 94-109. TREMBLAY, O. (1987). "Les maris paient. .. ", Justice, décembre.

WEITZMAN, L.J. (1985). The divorce revolution : The unexpected social and economic consequences for women and children in America. New York : Free Press. 